\title{
CXCL1 gene silencing inhibits HGC803 cell migration and invasion and acts as an independent prognostic factor for poor survival in gastric cancer
}

\author{
LIANG WANG ${ }^{*}$, CHANGHUA ZHANG ${ }^{*}$, JIANBO XU, HUI WU, \\ JIANJUN PENG, SHIRONG CAI and YULONG HE \\ Department of Gastrointestinal Surgery, First Affiliated Hospital, Sun Yat-sen University, \\ Guangzhou, Guangdong 510080, P.R. China
}

Received September 2, 2015; Accepted September 1, 2016

DOI: $10.3892 / \mathrm{mmr} .2016 .5843$

\begin{abstract}
Chemokine (C-X-C motif) ligand 1 (CXCL1) is essential in oncogenesis and development of malignant tumors. The present study aimed to investigate CXCL1 expression in promoting lymph node metastasis in gastric cancer patients. Human gastric cancer cell lines were employed to detect CXCL1 expression. HGC803 cell migration and cell invasion were detected using a wound healing assay and Transwell invasion assay, respectively. A total of 100 patients who underwent radical gastric resection with lymph node dissection in the First Affiliated Hospital of Sun Yat-Sen University (Guangzhou, China) between 2007 and 2008 were included. Expression of CXCL1 and lymphatic vessel density (LMVD) was determined by using immunohistochemistry (IHC), and their association with clinicopathological features and prognosis was investigated. Cox survival regression analysis was used to analyze overall survival of patients. Results indicated that CXCL1 protein was expressed in all of investigated gastric cancer cell lines. Silencing of the CXCL1 gene reduced migratory and invasive ability of HGC 803 cells. CXCL1 protein expression was detected by IHC in 41 patients (41\%), these were associated with advanced tumor-node-metastasis (TNM) stage, LMVD, tumor differentiation and poor survival. LMVD was positively correlated with advanced TNM stage, size of tumor, tumor differentiation and poor survival rate. Furthermore, it was observed that TNM stage, tumor differentiation and CXCL1 were independent prognostic factors in
\end{abstract}

Correspondence to: Dr Yulong He, Department of Gastrointestinal Surgery, First Affiliated Hospital, Sun Yat-sen University, 58 Zhongshan Second Road, Guangzhou, Guangdong 510080, P.R. China

E-mail: hylonggz@sina.com

*Contributed equally

Key words: CXCL1, gastric cancer, lymphangiogenesis, lymphatic vessel density the Cox survival regression analysis. Silencing of the CXCL1 gene inhibits HGC803 cell migration and invasion. The positive expression of CXCL1 is correlated with poor survival of gastric cancer patients and CXCL1 is an independent prognostic factor for gastric cancer.

\section{Introduction}

Gastric cancer is one of the most common malignant tumors in China. It has the highest morbidity and mortality rates of malignant tumors of the digestive system. Lymph node metastasis often represents the first step in the metastasis process. A previous study has indicated that the rate of metastatic lymph nodes was 5\% in early stages, and can reach 70\% in advanced gastric cancer (1).

Therefore, elucidation of the carcinomatous metastasis mechanism may enable development of more effective measures to suppress tumor metastasis, thus, improving the prognosis of patients with gastric cancer.

Previous studies have also demonstrated that the inflammatory response is an important feature of malignant neoplasms, and it is important in the genesis, growth and metastasis of tumors $(1,2)$. Chemokines are a large family, including numerous members, researchers have identified $>50$ types of chemokines (3). Chemokines mediate directional chemotactic movement during the inflammatory process as the predominant inflammatory factors. CXCLl belongs to the chemotactic superfamily and is expressed in neutrophils, macrophages, and epithelial cells. It has been observed in melanoma tumors, and are involved in the carcinogenesis of melanoma. CXCL1 specifically binds to the CXC chemokine receptor, CXC motif chemokine receptor 2 (CXCR2), which is a member of the $\mathrm{G}$ protein-coupled receptor family (4).

Previous studies have demonstrated CXCLl is important in the oncogenesis and development of malignant tumors. CXCL1 is upregulated in melanoma, ovarian, colorectal and bladder cancer, and other tumors (5-8). Numerous studies investigating the expression of CXCLl in the occurrence and development process of gastric cancer have produced consistent results (9-11). By comparing gene expression of the gastric cancer and adjacent tissues, it was observed that 
CXCLl is expressed in a high percentage of gastric cancer tissue samples, and it is associated with tumor stage and the prognosis of patients $(12,13)$.

A previous study (12) also observed a high expression of CXCR2 in gastric cancer tissue, and its positive correlation with TNM classification of gastric cancer and lymphatic vessel density (LMVD) indicates CXCLl and its receptor CXCR2 may be important in lymphatic metastasis. Furthermore, tumor cells may promote CXCLl expression in the endothelial cells of efferent lymphatic vessels, in order to promote a suitable microenvironment for tumor metastasis. Tumor cells may express CXCR2 and metastasize to lymphatic vessels under the attraction of CXCL1. Few studies have investigated the mechanism of the chemokine CXCLl/CXCR2 signaling pathway, thus, identifying specific underlying mechanisms may offer novel ideas for the treatment of gastric cancer with lymph node metastasis.

\section{Materials and methods}

Cell lines. The human gastric cancer cell lines, including HGC803, BGC823, AGS, MGC803, SGC7901 and MKN45 cells, were obtained from the Cell Bank of the Chinese Academy of Sciences (Shanghai, China) and cultured in Dulbecco's modified Eagle's medium (DMEM; Gibco; Thermo Fisher Scientific, Inc., Waltham, MA, USA) supplemented with $10 \%$ fetal bovine serum (FBS; Gibco; Thermo Fisher Scientific, Inc.) and streptomycin/penicillin $(100 \mathrm{mg} / \mathrm{ml}$ and $100 \mathrm{U} / \mathrm{ml}$, respectively) at $37^{\circ} \mathrm{C}$ in $5 \% \mathrm{CO}_{2}$ humidified atmosphere.

The normal human gastric cell line, GES-1 gastric epithelial cells were purchased from the Cell Bank of Chinese Academy of Sciences and used as the control for the experiments.

Patients. Informed consent was obtained from 100 patients (age, 18 to 75) diagnosed with gastric cancer at the First Affiliated Hospital of Sun Yat-sen University (Guangzhou, China) between 2007 to 2008 who were enrolled in the current study. Each patient had undergone gastrectomy with extended (D2) removal of regional lymph nodes. No patients had received adjuvant chemotherapy, radiation therapy or other biological therapy. The present study was approved by the Ethics Committee of the First Affiliated Hospital, Sun Yat-sen University (Guangzhou, China).

Exclusive criteria were as follows: i) $<18$ or $>75$ years of age; ii) patients with severe medical diseases or heart, lung, liver and kidney disorders; iii) patients with major postoperative complications; and iv) dissected lymph node number of $<15$. All the patients were followed until December 31, 2013. All specimens were fixed by formalin and embedded in paraffin. The clinicopathological characteristics and prognosis of patients were recorded in the hospital database.

Trial grouping in vivo and in vitro experiments. For the in vitro experiment, three groups were designed, including the CXCL1-siRNA group (with the siRNA to silence the CXCL1 gene), the HGC803 group (blank cells) and the CXCL1 stimuli group [human CXCL1 $\alpha$; obtained from R\&D Systems (Minneapolis, MN, USA)]. For the CXCL1-siRNA group, the HGC803 cells were treated with $0.5 \mu \mathrm{g}$ CXCL1 siRNA. For the CXCL-1 stimuli group, the HGC803 cells were treated with $10 \mu \mathrm{g}$ human CXCL1 $\alpha$.

For the in vivo experiment, the CXCL1 expression levels in 100 gastric cancer patients were analyzed using an immunohistochemistry assay, and overall survival analysis and correlation analysis were conducted.

Western blotting. The HGC803 cells were homogenized in ice-cold radioimmunoprecipitation lysis buffer (Sigma-Aldrich; Merck Millipore, Darmstadt, Germany) containing the cocktail of protease inhibitor (Roche Diagnostics, Basel, Switzerland). Lysates were centrifuged at $500 \times \mathrm{g}$ for $5 \mathrm{~min}$ at room temperature to obtain the protein. The extracted protein was quantified using a bicinchoninic acid protein assay kit (Beyotime Institute of Biotechnology, Haimen, China ). The quantified protein was separated by $15 \%$ SDS-PAGE $(0.2 \mu \mathrm{g}$ protein per well) and transferred to nitrocellulose membranes and blocked overnight at $4^{\circ} \mathrm{C}$ in $5 \%$ milk. The blocked membranes were incubated at $4^{\circ} \mathrm{C}$ overnight with the mouse anti-human CXCL1 monoclonal antibody (1:4,000; cat. no. 20326R; BD Biosciences, San Jose, CA, USA). Subsequently, the membranes were incubated with goat anti-rabbit IgG (1:2,000; cat. no. sc-2004; Santa Cruz Biotechnology, Inc., Dallas, TX, USA) and rabbit anti-mouse IgG (1:2,000; cat no. sc-358920; Santa Cruz Biotechnology, Inc.) at $37^{\circ} \mathrm{C}$ for $1 \mathrm{~h}$. Immunodetection was performed using the Amersham ECL Plus (GE Healthcare Life Sciences, Chalfont, UK). The blots were scanned and the pixel count and intensity of each band was quantified using the Scion image software (version 4.2.3.2; Scion Corporation, Frederick, MD, USA). The signals were normalized to GAPDH levels using a mouse anti-human GAPDH monoclonal antibody (cat. no. sc-365062; Santa Cruz Biotechnology, Inc.).

Wound healing assay. The HGC803 cells were plated in 6 -well plates at a density of $1 \times 10^{5}$ cells/well and grown to $\sim 80 \%$ confluency. The monolayer was scraped with a sterile $200 \mu \mathrm{l}$ pipette tip following removal of the culture medium. Subsequently, the culture was washed twice with serum-free medium. Cells were maintained in DMEM. Images of the scratched areas were captured at 0 and $24 \mathrm{~h}$ after wounding using computer-assisted optical microscopy. Cell migration was calculated as the percentage of cell coverage compared to the initial cell-free zone.

Transwell invasionassay. HGC803 cell invasion was evaluated using a Transwell chamber (Costar; Corning Incorporated, Corning, NY, USA) equipped with a Matrigel-coated filter membrane ( $8 \mu \mathrm{m}$ pores). Briefly, the filters were pre-coated with $0.5 \mu \mathrm{g}$ basement membrane proteins (Matrigel; BD Biosciences) and allowed to dry overnight at room temperature. Cells in FBS-free medium were seeded in the upper chambers, and lower wells contained medium with $10 \%$ FBS. Following incubation at $37^{\circ} \mathrm{C}$ for $24 \mathrm{~h}$, non-migratory cells on the upper side of the insert were removed with a cotton swab. Cells that had passed through the filter were fixed in methanol and stained with hematoxylin. For quantification, images were captured of six randomly selected fields on the lower side of the insert using computer-assisted optical microscopy. 
Table I. Clinicopathological associations between plasma CXCL1 expressions and LMVD in 100 gastric cancer patients.

\begin{tabular}{|c|c|c|c|c|c|c|}
\hline \multirow[b]{2}{*}{ Parameters } & \multirow[b]{2}{*}{$\mathrm{n}$} & \multicolumn{3}{|c|}{ Plasma CXCL1 } & \multicolumn{2}{|c|}{ LMVD } \\
\hline & & Negative & Positive & P-value & & P-value \\
\hline Gender & & & & 0.601 & & 0.970 \\
\hline Male & 65 & 39 & 26 & & $8.23 \pm 3.47$ & \\
\hline Female & 35 & 20 & 15 & & $9.31 \pm 2.23$ & \\
\hline Age (years) & & & 0.523 & & & 0.440 \\
\hline$<60$ & 40 & 25 & 15 & & $9.24 \pm 2.34$ & \\
\hline$\geq 60$ & 60 & 34 & 26 & & $9.03 \pm 1.68$ & \\
\hline Tumor size $(\mathrm{cm})$ & & & 0.290 & & & 0.003 \\
\hline$<4$ & 48 & 31 & 17 & & $5.55 \pm 2.56$ & \\
\hline$\geq 4$ & 52 & 28 & 24 & & $10.38 \pm 2.88$ & \\
\hline Location & & & 0.195 & & & 0.876 \\
\hline Proximal gastric cancer & 18 & 11 & 7 & & $11.53 \pm 3.23$ & \\
\hline Gastric body cancer & 29 & 18 & 11 & & $9.34 \pm 1.38$ & \\
\hline Distal gastric cancer & 53 & 30 & 23 & & $8.78 \pm 2.56$ & \\
\hline $\mathrm{T}$ classification & & & 0.031 & & & 0.002 \\
\hline $\mathrm{T} 1 / \mathrm{T} 2$ & 36 & 31 & 5 & & $4.56 \pm 1.23$ & \\
\hline $\mathrm{T} 3 / \mathrm{T} 4$ & 64 & 28 & 36 & & $10.23 \pm 2.31$ & \\
\hline WHO classification & & & 0.062 & & & 0.890 \\
\hline Adenocarcinoma & 71 & 43 & 28 & & $8.45 \pm 2.35$ & \\
\hline Mucinous adenocarcinoma & 18 & 10 & 8 & & $9.56 \pm 1.23$ & \\
\hline Signet ring cell carcinoma & 11 & 6 & 5 & & $10.12 \pm 3.78$ & \\
\hline Differentiated degree & & & $<0.001$ & & & 0.04 \\
\hline High/middle differentiation & 38 & 30 & 8 & & $6.03 \pm 1.39$ & \\
\hline Low/no differentiation & 62 & 29 & 33 & & $11.86 \pm 3.67$ & \\
\hline $\mathrm{N}$ classification & & & $<0.001$ & & & 0.02 \\
\hline Negative & 42 & 34 & 9 & & $5.37 \pm 2.11$ & \\
\hline Positive & 58 & 25 & 32 & & $12.04 \pm 3.88$ & \\
\hline TNM classification & & & $<0.001$ & & & $<0.001$ \\
\hline $\mathrm{I} / \mathrm{II}$ & 43 & 33 & 10 & & $4.06 \pm 1.56$ & \\
\hline III/IV & 57 & 26 & 31 & & $12.23 \pm 3.31$ & \\
\hline
\end{tabular}

CXCL1, chemokine (C-X-C motif) ligand 1; LMVD, lymphatic vessel density; WHO, World Health Organization; TNM, tumor-node-metastasis.

Immunohistochemistry. The tissues were frozen and then sliced into $4-\mu \mathrm{m}$ sections. In the sections, cell nuclei or cytoplasm stained yellow to yellow-brown were considered as positive. The cells were incubated with rabbit anti-human CXCL1 monoclonal antibody (1:3,000 dilution; cat. no. sc-2778; Santa Cruz Biotechnology, Inc.) and mouse anti-human D2-40 monoclonal antibody (1:3,000 dilution; cat. no. 182410; Invitrogen; Thermo Fisher Scientifc, Inc.) at $37^{\circ} \mathrm{C}$ for $2 \mathrm{~h}$. Content determination results were analyzed by HMIAS-2000 automatic medical color image analysis system (Qianping Image Technology Co., Ltd., Wuhan, China). Sections were observed using light microscopy, 5 randomly selected high power fields were observed and grey values of specimens were obtained following image segmentation, editing and statistics scoring criteria. Stained area: 0 , stained cell area of $\leq 10 \%$ of overall cell area; $1,11-25 \% ; 2,26-50 \% ; 3,51-75 \%$;
4, $>75 \%$. Staining intensity: 0, without staining; 1 , yellow or light brown; 2, brown; 3, dark brown. The total score of each section was defined as the product of the stained area score and the staining intensity score, a score of $\leq 3$ was negative, while 3-12 was positive.

Determination of lymphatic vessel density in gastric cancer and peri-carcinoma region. Immunohistochemical staining (as described above) with the D2-40 monoclonal antibody (1:200) was used for detection of lymphatic vessels. LMVD in gastric cancer and the peri-carcinoma region was determined by counting lymph vessels. D2-40-positive lymphatic vessel endothelial cells were observed by microscope and appeared brown. Microlymphatic vessels were counted by Weidtler calculation standard, first, a comprehensive observation of the section was conducted at a lower magnification (x100) to 
observe new vessels, the most densely populated areas were determined to be 'hot spots', which were subsequently stained purple (by the hematoxylin-eosin staining method) $(6,8)$ and observed at the single cell and cell cluster level at high magnification (x200). The mean value was determined to be the section LMVD value.

Statistical analysis. Analysis of data was conducted using SPSS 17.0 (SPSS, Inc., Chicago, IL, USA), the association between expression of CXCL1, D2-40 and clinicopathological features were investigated by $\chi^{2}$ test. The patient 5 -year survival rate was determined by Kaplan-Meier analysis. The Cox regression model was established to analyze the peaks of metastasis and relapse, and the life curves of patients. The group data were presented as the mean \pm standard error of the mean. $\mathrm{P}<0.05$ was considered to indicate a statistically significant difference.

\section{Results}

CXCL1 protein expression was increased in gastric cancer cell lines. In order to investigate the association between CXCL1 protein expression and the gastric cancer cell lines, protein expression was examined by western blotting (Fig. 1A). The results indicated that the CXCL1 protein was expressed in all the gastric cancer cell lines, and the levels were significantly higher compared to the levels in the GES-1 normal cell line (Fig. 1B; P<0.01). Furthermore, it was observed that the HGC803 cell line expressed the highest level of CXCL1. Thus, in the subsequent in vitro experiments, the HGC803 cell line was used.

Silencing of the CXCL1 gene reduced migratory and invasive ability of HGC803 cells. To assess the migratory ability of CXCL1 siRNA silenced HGC803 cells, a wound healing and a Transwell migration assay were used. The Transwell migration assay demonstrated that the number of migrated cells in the HGC803 control group were significantly higher when compared with the CXCL1 stimuli cells (Fig. 2). Few invaded cells were observed in the CXCL1-siRNA groups compared with the HGC803 control group (Fig. 2).

The results demonstrated that the wounds in the CXCL1 stimuli group were almost closed at the $24 \mathrm{~h}$ time point, yet the wounds in the HGC803 control group remained observable (Fig. 3). Notably, the wounds in the CXCL1-siRNA group were enlarged after $24 \mathrm{~h}$ treatment (Fig. 3). These results suggest knockdown of CXCL1 suppressed cell invasion.

Clinical pathology data for gastric cancer patients. In the present study, tumor size, tumor location, classification and differentiation were observed. The clinicopathological correlations of plasma CXCL1 expression and lymphatic vessel density are presented in Table I.

CXCL1 expression in gastric tumor tissues. The present study included a total of 100 patients, and the expression levels of CXCL1 and the clinicopathological characteristics are presented in Table I. The rate of patients with positive CXCL1 expression is $41 \%$. Expression of CXCL1 has no marked association with gender, age, tumor location or tumor diameter,
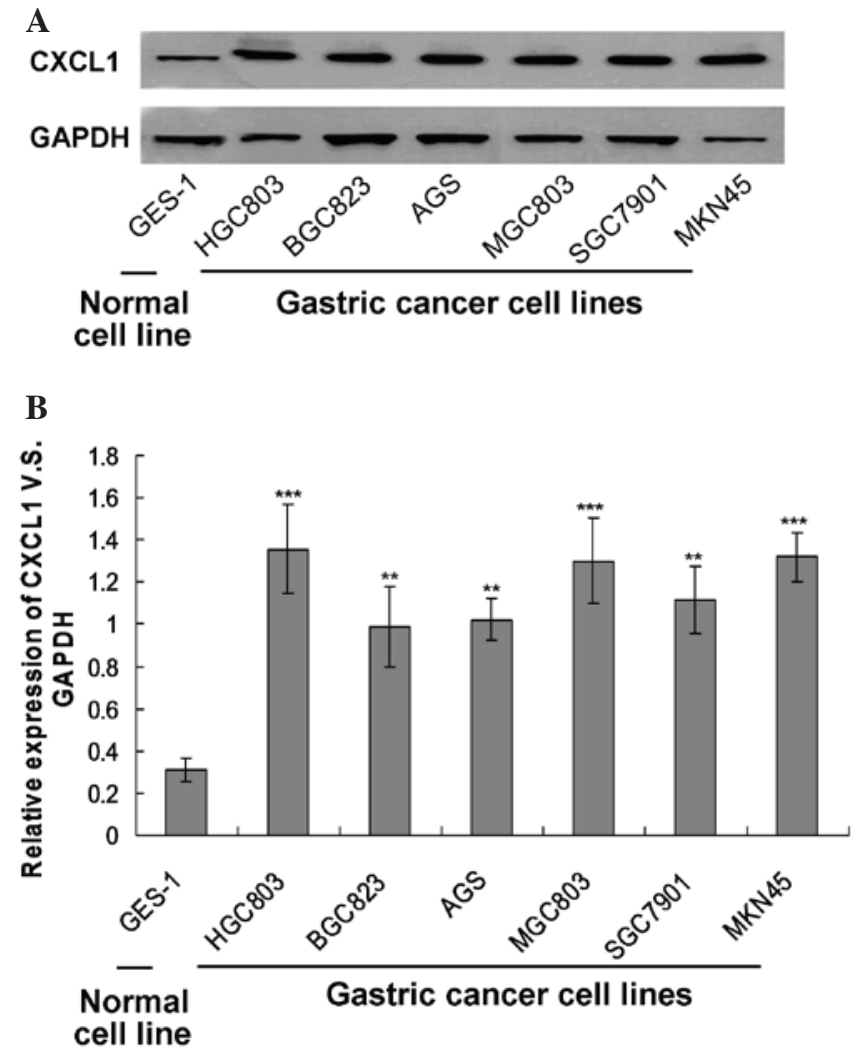

Figure 1. CXCL1 protein expression in normal and gastric cancer cell lines. (A) Western blot assay for the CXCL1 protein expression. (B) Statistical analysis of CXCL1 expression. ${ }^{* *} \mathrm{P}<0.01$ and ${ }^{* * *} \mathrm{P}<0.001$ vs. the normal cell line. CXCL1, chemokine (C-X-C motif) ligand 1.

however, it is closely associated with degree of tumor differentiation, lymph node metastasis and TNM classification $(\mathrm{P}<0.05)$. Immunohistochemical staining demonstrated that expression of CXCL1 was detected in the cell cytoplasm (Fig. 4). The CXCL1 positive group had significantly higher TNM stage and poorer pathological differentiation.

Expression of D2-40 in gastric cancer and the peri-carcinoma region. D2-40 positive staining of lymphatic vessels in the normal gastric mucosa were located in the deeper layers of the mucosa and close to submucosal muscular layer, with larger lumen and thicker walls. New lymphatic vessels were only observed between the inherent glands of the mucosa lamina propria, as presented in Fig. 4. New D2-40 positive staining of lymphatic vessels was yellow brown, located in the tumor stroma and no red blood cells observed. At low magnification (x100), five intense staining areas were selected and 100 positive cells in each field were observed at high magnification. The average percentage of positive cells from the five areas indicate the LMVD results and are presented in Table I.

Correlation between LMVD and pathological biological characteristics. LMVD positively correlated with gastric cancer lesion size, degree of differentiation, clinical TNM stage and depth of invasion (Fig. $5 ; \mathrm{P}<0.05$ ), while there is no notable correlation between lymphatic vessel density (LMVD) and age, gender, WHO classification or tumor location. 

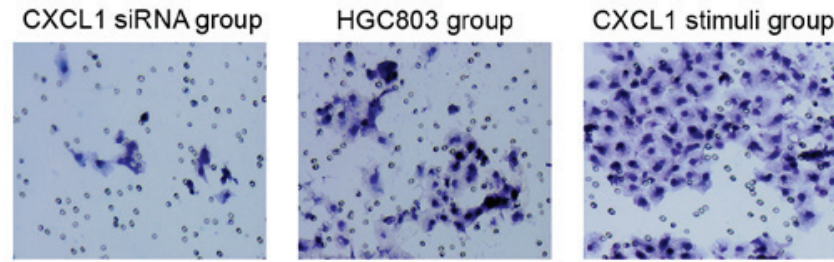

Figure 2. CXCL1 gene silencing inhibited HGC803 cell invasion Representative images of the invaded cells (stained with hematoxylin) selected from three experiments are presented above. Migrated cells were counted under a microscope. CXCL1, chemokine (C-X-C motif) ligand 1; siRNA, small interfering RNA.

CXCL1 protein expression reflects gastric cancer survival rate. The 5-year survival rate was determined by Kaplan-Meier analysis. CXCL1 positive group ( $\mathrm{n}=41)$ exhibited poorer survival rates in 5 years than the negative group $(n=59)$ as presented in Fig. $6(\mathrm{P}=0.002)$. The total mean survival time of the negative group was 40 months, with that of positive group typically 23.2 months. This is a statistically significant difference.

Analysis of independent prognostic factor of patients with radical gastrectomy of gastric cancer. The Cox regression model was established to analyze the peaks of metastasis and relapse, and the life curves of patients (Table II). Following multivariate analysis, CXCL1, differentiation degree, TNM stage and LMVD may be used as independent prognostic factors.

\section{Discussion}

Lymphatic metastasis is a major mechanism of metastasis of malignant tumors, and key in determination of prognosis (14). Lymphangiogenes is a process of developing new capillary lymphatic vessels by budding and further differentiation. Vasculature are at a stable state following maturity, new blood vessels or the lymphatic vessels are formed only in certain conditions, such as embryonic development, inflammation, wound healing, and tumors. No development of lymph node metastases is generally due to lack of lymphatic vessels at the tumor site in earlier stages of the neoplastic process.

Chemokines are members of the cytokine superfamily, which are small molecule proteins that induce chemotaxis (15). According to different positions of four conservative cysteine residues in its $\mathrm{N}$ terminal region, chemokines may be divided into A, 3, Y and S chemokine subfamilies. A subfamily (also called CXC subfamily) includes IL-8, CXCL1, CXCL2, and CXCL3. Via interaction with their receptors, chemokines induce chemotactic migration of target cells, enhance the adhesive capacity of endothelial cells to target cells, and participate in cellular functions, including proliferation, apoptosis, invasion and differentiation. Previous studies have demonstrated that chemokines are involved in pathogenesis, progression, and metastasis of carcinoma $(16,17)$. For example, IL-8 may regulate tumor angiogenesis, induce release of proteolytic enzymes and stimulate the proliferation of tumor cells by degradation of the extracellular matrix and basement membrane, and promote the invasion and metastasis of gastric cancer via autocrine or paracrine modes of action. At present,

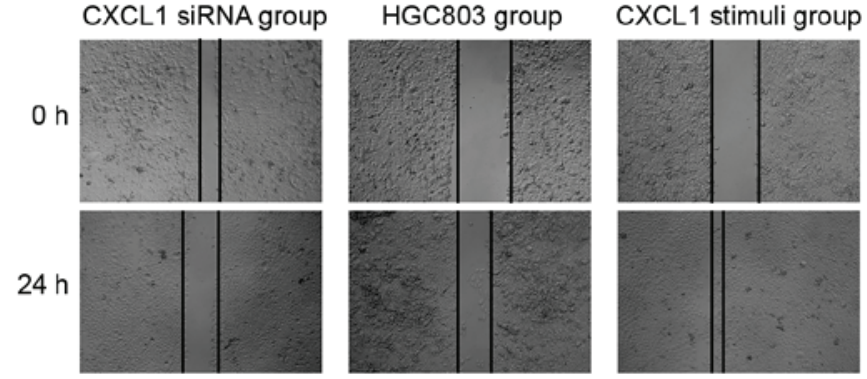

Figure 3. CXCL1 gene silencing inhibited HGC803 cell migration. Wound healing was examined in stable cells at 0 and $24 \mathrm{~h}$ after wound formation. Representative images from three experiments are presented. CXCL1, chemokine (C-X-C motif) ligand 1; siRNA, small interfering RNA.
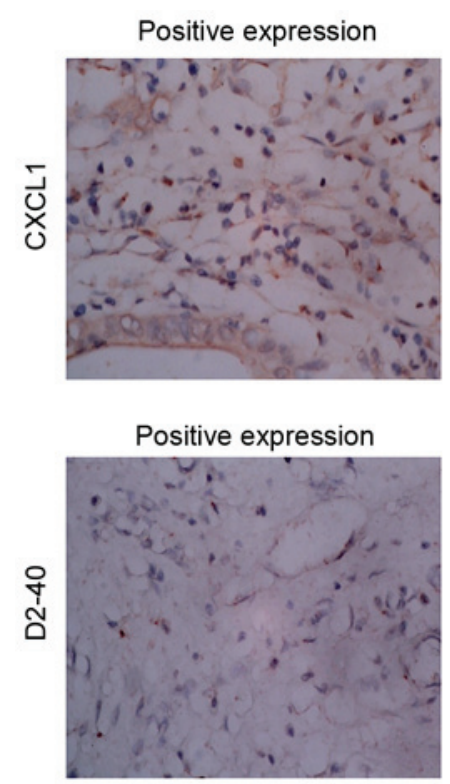

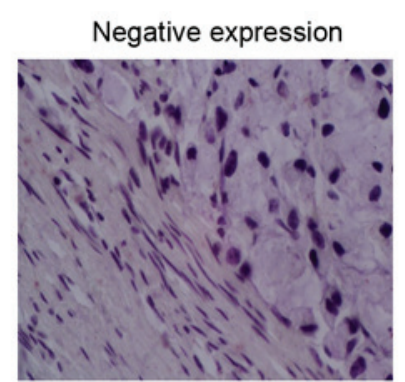

Negative expression

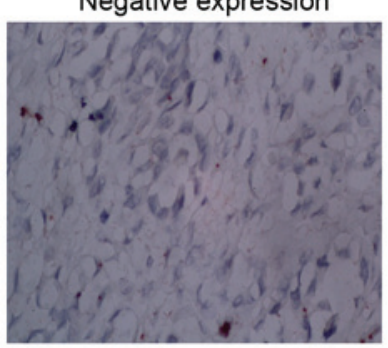

Figure 4. CXCL1 and D2-40 expression in gastric tumor tissues. CXCL1, chemiokine (C-X-C motif) ligand 1.

there has been no investigation into the association between CXCL1 expression and LMVD.

The present study analyzed the association between CXCL1 expression and LMVD in clinical specimens of gastric cancer tissue and clinicopathological features. Kaplan-Meier analysis and the Cox regression model were used to analyze the association between CXCL1 expression, LMVD and prognosis in gastric cancer. In addition, the relevant independent prognostic factors were investigated. Experimental results indicate that the CXCL1 positive expression in patients with gastric cancer have higher LMVD, which is positively correlated with tumor invasion, lymph node metastasis, TNM stage and the degree of differentiation, indicating the CXCL1 may participate in the pathogenesis and development of gastric cancer. This finding was consistent with our previous study (12). A number of previous studies (15-17) have demonstrated that CXCL1 is overexpressed in gastric cancer tissue, which is associated with infiltration depth, and lymph node involvement, thus, affecting the prognosis of patients, and that CXCL1 may promote lymphatic and blood metastasis of gastric carcinoma. The current study also observed that the D2-40-positive staining 
Table II. Results from multivariate analysis of Cox regression models.

\begin{tabular}{lccr}
\hline Parameters & P-value & Relative risk & 95\% CI \\
\hline Differentiation degree & 0.04 & 2.204 & $0.818-3.048$ \\
TNM classification & 0.005 & 2.236 & $1.468-4.379$ \\
CXCL1 & 0.003 & 1.904 & $1.406-4.148$ \\
LMVD & 0.02 & 1.616 & $1.168-3.779$ \\
\hline
\end{tabular}

CI, confidence interval; TNM, tumor-node-metastasis; CXCL1, chemokine (C-X-C motif) ligand 1; LMVD, lymphatic vessel density.
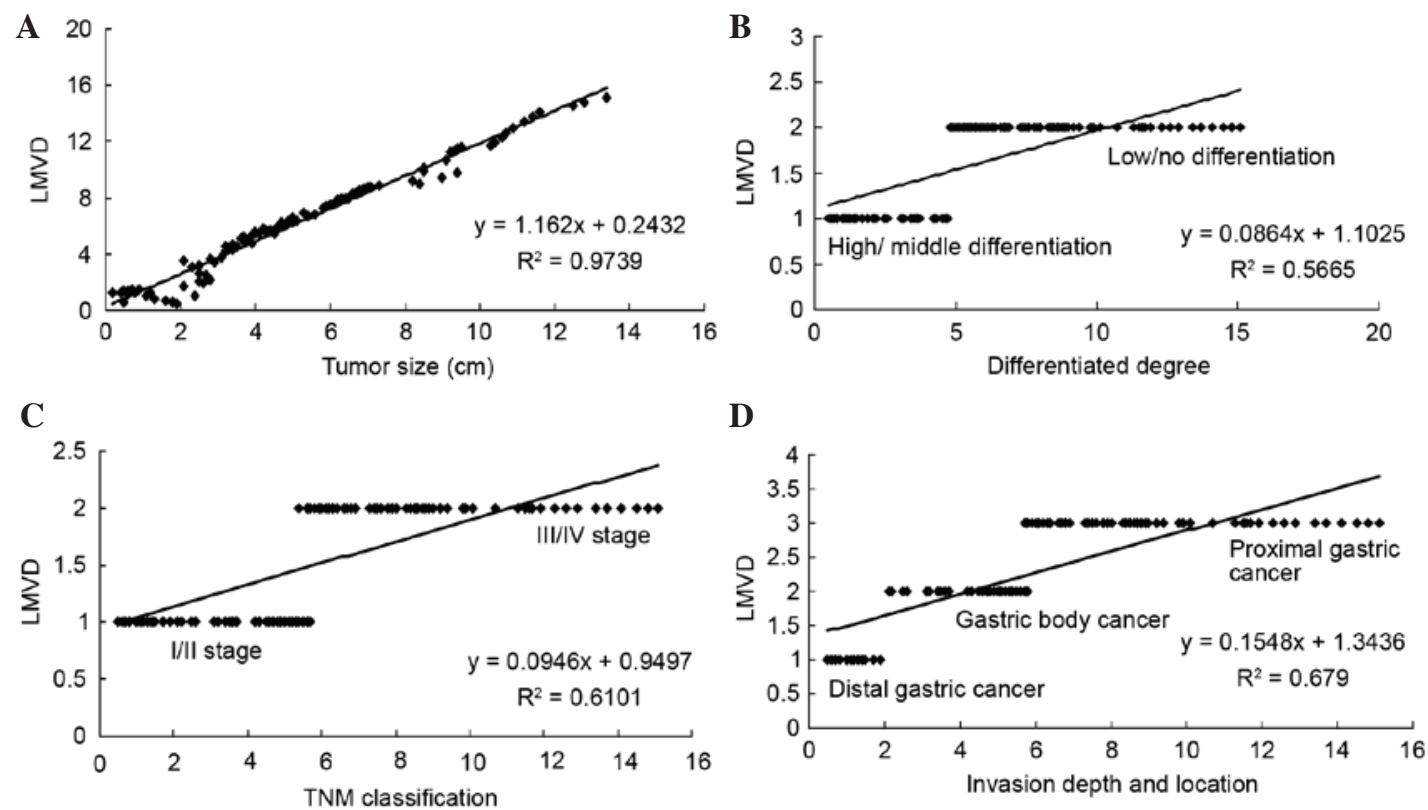

Figure 5. Correlation analysis for LMVD and the other parameters. (A) Correlation analysis between LMVD and (A) tumor size, (B) degree of differentiation, (C) TNM classification and (D) invasion depth and location. LMVD, lymphatic vessel density; TNM, tumor-node-metastasis.

was predominantly localized in lymphatic endothelial cells, while no expression of D2-40 was observed in tumor cells. LMVD was higher in poorly differentiated carcinomas than in well or moderately differentiated cases. LMVD in gastric cancer with lymph node metastasis was significantly higher than in those without lymph node metastasis. Under similar conditions, LMVD in gastric cancer with positive expression of CXCL1 is significantly higher than in gastric cancer with negative expression of CXCL1. Significant positive correlation was observed between expression of CXCL1 and LMVD in gastric cancer tissues $(\mathrm{P}<0.05)$. These results indicate LMVD was closely associated with gastric cancer, and has a significant correlation with the size of carcinoma, classification, clinical TNM stages, depth of invasion and lymph node metastasis. Thus, LMVD may be used as an indicator for differential diagnosis and determination of malignancy of gastric cancer, and may aid prediction of prognosis and treatment decisions for patients with gastric cancer.

Furthermore, results of the Kaplan-Meier survival curves demonstrated that the expression of CXCL1 and LMVD may worsen prognosis as patients with positive expression exhibit a significantly reduced 5-year survival rate than those with negative expression. The experimental results obtained are

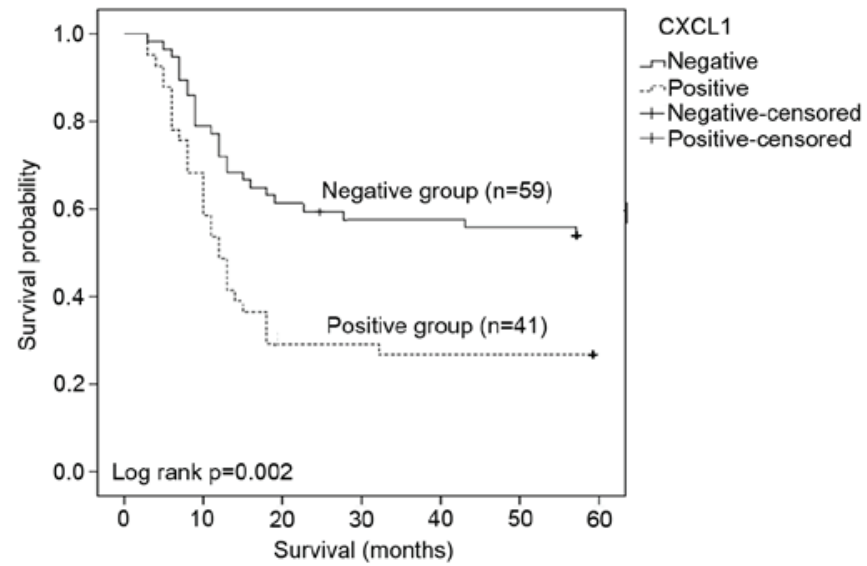

Figure 6. Kaplan-Meier survival curves of two groups of gastric cancer patients defined by CXCL1 expression levels. CXCL1, chemokine (C-X-C motif) ligand 1 .

consistent with results of our previous study (12). Results of the further Cox survival analysis indicated that expression of CXCL1, TNM stage and differentiation degree and LMVD all can be used as independent prognostic factors. 
The results of the present study suggest that high expression of CXCL1 in gastric cancer tissue was closely associated with tumor differentiation and staging, indicating that CXCL1 may be a potential tumor marker. At the earlier stages of the neoplastic process, CXCL1 may affect the tumor microenvironment and then promote the growth of tumor cells and angiogenesis, suggesting that CXCL1 may also be used as a biomarker for monitoring clinical effect. The current study identified the association between expression of CXCL1 and gastric cancer growth, prognosis and the clinicopathological features, which may aid elucidation of the underlying mechanism of the CXCL1/CXCR2 signaling pathways and have potential for therapeutic agent intervention.

In conclusion, CXCL1 gene silencing inhibited the migration and invasion of HGC803 cells. The positive expression of CXCL1 is correlated with advanced TNM stage, LMVD, tumor differentiation, lymph node metastasis and poor survival. LMVD was correlated with advanced TNM stage, size of tumor, lymph node metastasis, tumor differentiation and poor survival. CXCL1 may be an independent prognostic factor for gastric cancer.

\section{Acknowledgements}

The present study was supported by the National Natural Science Fund (grant no. 81272637).

\section{References}

1. Hua D, Shen L, Xu L, Jiang Z, Zhou Y, Yue A, Zou S, Cheng Z and $\mathrm{Wu}$ S: Polypeptide N-acetylgalactosaminyltransferase 2 regulates cellular metastasis-associated behavior in gastric cancer. Int J Mol Med 30: 1267-1274, 2012.

2. Bernal C, Aguayo F, Villarroel C, Vargas M, Díaz I, Ossandon FJ, Santibáñez E, Palma M, Aravena E, Barrientos C and Corvalan AH: Reprimo as a potential biomarker for early detection in gastric cancer. Clin Cancer Res 14: 6264-6269, 2008.

3. Dhawan P and Richmond A: Role of CXCL1 in tumorigenesis of melanoma. J Leukoc Biol 72: 9-18, 2002.

4. Ravindran A, Sawant KV, Sarmiento J, Navarro J and Rajarathnam K: Chemokine CXCL1 dimer is a potent agonist for the CXCR2 receptor. J Biol Chem 288: 12244-12252, 2013.

5. Yonemura Y, Fonseca L, Tsugawa K, Ninomiya I, Matsumoto H, Sugiyama K, Ohoyama S, Fushida S, Kimura H and Miyazaki I: Prediction of lymph node metastasis and prognosis from the assay of the expression of proliferating cell nuclear antigen and DNA ploidy in gastric cancer. Oncology 51: 251-257, 1994.
6. Wallace AE, Sales KJ, Catalano RD, Anderson RA, Williams AR, Wilson MR, Schwarze J, Wang H, Rossi AG and Jabbour HN: Prostaglandin F2alpha-F-prostanoid receptor signaling promotes neutrophil chemotaxis via chemokine (C-X-C motif) ligand 1 in endometrial adenocarcinoma. Cancer Res 69: 5726-5733, 2009.

7. Yang G, Rosen DG, Zhang Z, Bast RC Jr, Mills GB, Colacino JA, Mercado-Uribe I and Liu J: The chemokine growth-regulated oncogene 1 (Gro-1) links RAS signaling to the senescence of stromal fibroblasts and ovarian tumorigenesis. Proc Natl Acad Sci USA 103: 16472-16477, 2006.

8. Cheng WL, Wang CS, Huang YH, Liang Y, Lin PY, Hsueh C, Wu YC, Chen WJ, Yu CJ, Lin SR and Lin KH: Overexpression of a secretory leukocyte protease inhibitor in human gastric cancer. Int J Cancer 123: 1787-1796, 2008

9. Wu CC, Chien KY, Tsang NM, Chang KP, Hao SP, Tsao CH, Chang YS and Yu JS: Cancer cell-secreted proteomes as a basis for searching potential tumor markers: Nasopharyngeal carcinoma as a model. Proteomics 5: 3173-3182, 2005.

10. Goulding H, Pinder S, Cannon P, Pearson D, Nicholson R, Snead D, Bell J, Elston CW, Robertson JF, Blamey RW, et al: A new immunohistochemical antibody for the assessment of estrogen receptor status on routine formalin-fixed tissue samples. Hum Pathol 26: 291-294, 1995.

11. Kawanishi H, Matsui Y, Ito M, Watanabe J, Takahashi T, Nishizawa $K$, Nishiyama $H$, Kamoto T, Mikami Y, Tanaka Y, et al: Secreted CXCL1 is a potential mediator and marker of the tumor invasion of bladder cancer. Clin Cancer Res 14: 2579-2587, 2008

12. Eck M, Schmausser B, Scheller K, Brändlein S and Müller-Hermelink HK: Pleiotropic effects of CXC chemokines in gastric carcinoma: Differences in CXCL8 and CXCL1 expression between diffuse and intestinal types of gastric carcinoma. Clin Exp Immunol 134: 508-515, 2003.

13. Singh S, Sadanandam A, Nannuru KC, Varney ML, Mayer-Ezell R, Bond R and Singh RK: Small-molecue antagonists for CXCR2 and CXCR1 inhibit human melanoma growth by decreasing tumor cell proliferation, survival, and angiogenesis. Clin Cancer Res 15: 2380-2386, 2009.

14. Kitadai Y, Haruma K, Mukaida N, Ohmoto Y, Matsutani N, Yasui W, Yamamoto S, Sumii K, Kajiyama G, Fidler IJ and Tahara E: Regulation of disease-progression genes in human gastric carcinoma cells by interleukin 8. Clin Cancer Res 6: 2735-2740, 2000.

15. Zhi Y, Lu H, Duan Y, Sun W, Guan G, Dong Q and Yang C: Involvement of the nuclear factor-kB signaling pathway in the regulation of CXC chemokine receptor- 4 expression in neuroblastoma cells induced by tumor necrosis factor- $\alpha$. Int J Mol Med 35: 349-357, 2015.

16. Salazar N, Castellan M, Shirodkar SS and Lokeshwar BL: Chemokines and chemokine receptors as promoters of prostate cancer growth and progression. Crit Rev Eukaryot Gene Expr 23: 77-91, 2013.

17. Wakabayashi S, Yamaguchi K, Kumakura S, Murakami T, Someya A, Kajiyama Y, Nagaoka I and Inada E: Effects of anesthesia with sevoflurane and proporol on the cytokine/chemokine production at the airway epithelium during esophagectomy. Int J Mol Med 34: 137-144, 2014. 